\title{
Fars Samann* and Thomas Schanze Finding an optimal dictionary of different wavelet types using sparse modeling to denoise ECG signal
}

\begin{abstract}
Sparse signal modeling often reconstructs a signal with few atoms from a pre-defined dictionary. Hence the choice of wavelet dictionary that represents the sparsity of the target signal is crucial in sparse modeling approach. The challenge of finding an optimal dictionary of different wavelet types using sparse denoising model (SDM) to denoise ECG signal is investigated in this work. A method of finding an optimal wavelet dictionary from a set of orthogonal wavelet sub-dictionaries by the means of the best correlation with ECG signal, is developed. The highly correlated sub-dictionaries from three wavelet dictionaries, namely daubechies, symlets, coiflets and discrete cosine transform are combined to construct an overcomplete dictionary. The weight of Akaike's information criterion and the signal-to-noise ratio improvement are considered as a criterion to evaluate the performance of the proposed SDM. The results indicate that multi-wavelet dictionary of different types is highly sparse and efficient in denoising the target signal, e.g., ECG.
\end{abstract}

Keywords: Sparse denoising model (SDM), Multi-wavelet type dictionary (MWD), Matching pursuit algorithm.

https://doi.org/10.1515/cdbme-2021-2032

\section{Introduction}

It is important to denoise a biomedical signal-such as the electrocardiogram (ECG) - to allow a valid diagnosis of

*Corresponding author: Fars Samann: Technische Hochschule Mittelhessen (THM), FB Life Science Engineering (LSE), Institut für Biomedizinische Technik (IBMT), Wiesenstr. 14, 35390 Gießen, Germany, e-mail: fars.esmat.fathel.samann@lse.thm.de, Department of Biomedical Engineering, University of Duhok, 42001 Duhok, Kurdistan Region-Iraq

Thomas Schanze, Technische Hochschule Mittelhessen (THM), FB Life Science Engineering (LSE), Institut für Biomedizinische Technik (IBMT), Wiesenstr. 14, 35390 Gießen, Germany, e-mail: thomas.schanze@Ise.thm.de cardiovascular diseases. The ECG signal is naturally sparse, since it consists of three main signal parts: the QRS complex, $\mathrm{P}$-wave and T-wave. Besides, it contains different types of noise, e.g., powerline interference, baseline wander, gaussian white noise and motion artifacts [1]. Several methods of sparse modeling and representation have been developed and applied for compressing or denoising applications designed for different type of signals, e.g., biomedical signals and images [2]. However, to achieve an adequate sparse model for the target signal, it is crucial to establish a dictionary composed of atoms which sufficiently and efficiently represent the main features contained in the target signal. Therefore, many of dictionaries based on wavelets, curvelets, contourlets, etc. have been proposed for various applications.

In the literature, many denoising sparse modeling approaches of ECG signal have been proposed for ECG signal using pre-defined dictionaries obtained by analytical and mathematical modelling of the data, e.g., gaussian dictionaries [2], different mother wavelets (e.g., the coif4 and sym4) [3]. Although these approaches demonstrated useful results, the selection of these dictionaries were mainly based on the similarity between the atoms and the dominated waveform component of ECG signal (e.g., similarity between coiflet4 waveform with QRS complex waveform). This may lead to deficiency in representing other parts of ECG signal, i.e., low frequency components like P-or T-wave.

In this work, we describe an efficient method to construct an overcomplete dictionary from sub-dictionaries of different types of wavelets, plus discrete cosine transform (DCT) dictionary. This method is based on selecting the types of wavelet sub-dictionaries by the means of the best correlation and the sparsity in representing the ECG signal. we tend to answer the following questions: i) Which type of wavelet dictionary is suitable for a given signal? ii) How to estimate the optimal number of non-zero coefficients in the sparsity matrix? For this, Akaike's information criterion and signal-tonoise ratio analysis are used to find the optimal dictionary and thus the most appropriate elementary dictionary signals, i.e., the atoms. 


\section{Materials and Methods}

\subsection{ECG segment preparation}

The database of CPSC 2018 [4] is considered to establish a dataset of 200 QRS complex aligned ECG segments from 20 healthy patients (segment length $m=396$ samples and $f_{\text {sampling }}=500 \mathrm{~Hz}$ ). All the ECG segments are filtered by a third-order low-pass Butterworth filter with $f_{\text {cutoff }}=35 \mathrm{~Hz}$.

\subsection{Sparse denoising model}

The aim of sparse modeling is to represent sparse signals from small number of elementary dictionary signals (atoms). We consider overcomplete dictionary $\mathbf{D} \in \mathbb{R}^{m \times L}$, where the columns represent the dictionary atoms and the length of atoms is $m$, and $L>m$ is the number of atoms. Assume, that a number of $n$ noisy ECG segments can be presented as a single matrix $\mathbf{Y}=\left[\mathbf{y}_{1} \mathbf{y}_{2} \ldots \mathbf{y}_{\mathrm{n}}\right] \in \mathbb{R}^{m \times n}$, where each vector $\mathbf{y}_{\mathrm{i}} \in \mathbb{R}^{m \times 1}$ can be expressed as a linear combination of the dictionary atoms,

$$
\mathbf{y}_{\mathrm{i}}=\mathrm{D} \boldsymbol{\alpha}_{\mathrm{i}}
$$

where $\boldsymbol{\alpha}_{\mathrm{i}} \in \mathbb{R}^{L \times 1}$ is sparsity vector coefficients. Here we tend to represent the noisy ECG segment $\mathbf{y}_{\mathbf{i}}$ by few numbers of atoms to reconstruct noise free signal $\mathbf{x}_{\mathrm{i}}$. This means eq. (1) can be rewritten as $\hat{\mathbf{x}}_{\mathrm{i}} \approx \mathbf{D} \boldsymbol{\alpha}_{\mathrm{i}}$. Therefore, we apply a sparsity-constrained to find sparsity vector $\widehat{\boldsymbol{\alpha}}_{\mathrm{i}}$ with limited number of non-zeros coefficient $c$ as follow [2],

$$
\widehat{\boldsymbol{\alpha}}_{\mathrm{i}}=\arg \min _{\boldsymbol{\alpha}_{\mathbf{i}}} 1 / 2\left\|\mathbf{y}_{\mathbf{i}}-\mathbf{D} \boldsymbol{\alpha}_{\mathrm{i}}\right\|_{2} \text { s.t. }\left\|\boldsymbol{\alpha}_{\mathbf{i}}\right\|_{0} \leq c .
$$

The matching pursuit algorithm is considered to find the unique set of coefficients in sparsity vector $\widehat{\boldsymbol{\alpha}}_{\mathrm{i}}$ that represent the signal in a particular basis $\mathbf{D}$.

\subsection{Mother wavelet dictionary}

In general, the mother wavelet is basically a function of zero average value with specific time course which is controlled by two parameters, scaling factor $a$ and shifting factor $b$. This can be used to define an orthogonal basis [3],

$$
\psi_{a b}(t)=\frac{1}{\sqrt{a}} \psi\left(\frac{t-b}{a}\right) .
$$

In this work, we consider three families of mother wavelet dictionaries, namely daubechies $(\mathrm{dbN})$, symlets $(\mathrm{symN})$ and coiflets (coifN). Each of these dictionaries is consisted of a mother wavelet of level $=3$ with different $N$ vanishing moment sub-dictionaries (see Figure 1), and each sub-dictionary is consisted of level 1, 2, 3 and 3s (scaling). Each level has different shifting factor $a$ and number of shifted atoms as shown in table 1 . The number of shifted atoms for each level is calculated, where the atom length $m=396$,

$$
\text { No. of shifted atoms }=\text { round }\left(\frac{m}{b}\right) \text {. }
$$

Table 1: Number of shifted atoms for each level of sub-dictionary.

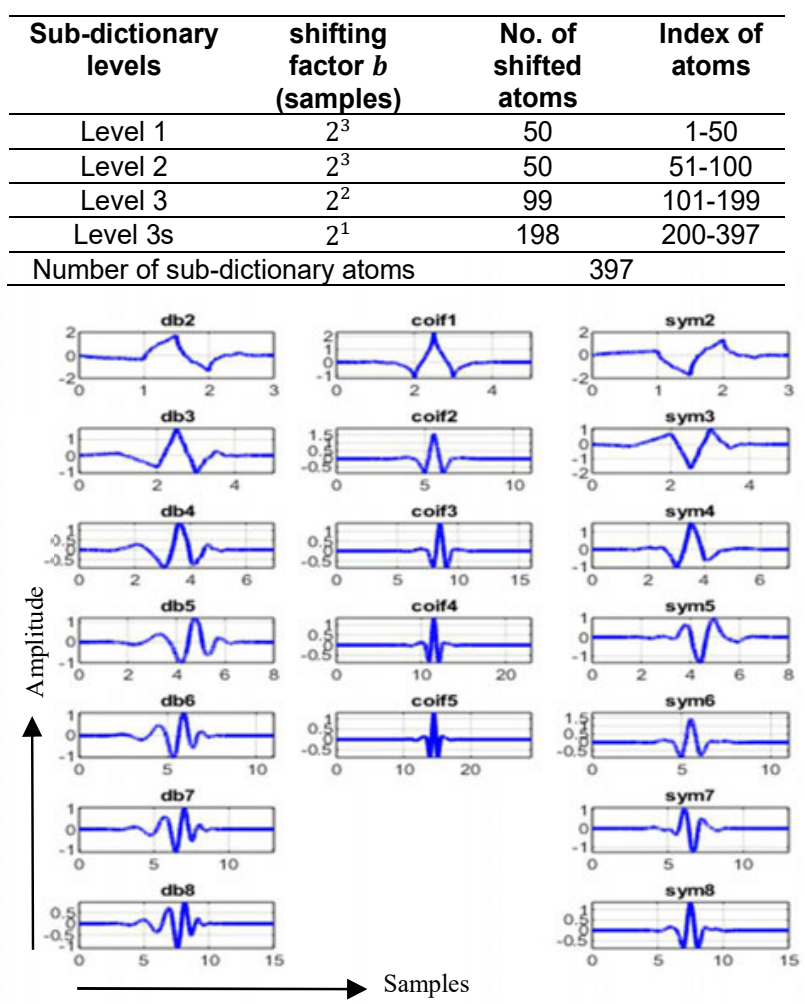

Figure 1: The waveform of an atom for different $N$ vanishing moment sub-dictionaries, starting with $N$ which are available in MATLAB (e.g., db2, coif1 \& sym2), in case of different mother wavelet dictionaries (left) dbN, (middle) coifN, (right) symN.

\subsection{Finding the optimal wavelet}

The matching pursuit toolbox in MATLAB2018a is considered in constructing the dictionary $\mathbf{D}$ and finding the sparsity matrix $\widehat{\boldsymbol{\alpha}}_{\mathrm{i}}$. The method of finding the optimal wavelet dictionary is developed as follows: we assume a noise free dataset of $n$ QRS aligned ECG segments of length $m$ each, $\mathbf{X}=\left[\mathbf{x}_{1} \mathbf{x}_{2} \ldots \mathbf{x}_{\mathrm{n}}\right] \in \mathbb{R}^{m \times n}$ :

1. Set $\mathbf{D}=\mathrm{dbN}$, then repeat with $\mathbf{D}=\operatorname{symN}$ and coifN.

2. Set number of non-zeros coefficient $c=m$.

3. For $i=1: n$ do

4. Find $\widehat{\boldsymbol{\alpha}}_{\mathrm{i}}=\arg \min _{\boldsymbol{\alpha}_{\mathrm{i}}} 1 / 2\left\|\boldsymbol{x}_{\mathrm{i}}-\mathbf{D} \boldsymbol{\alpha}_{\mathrm{i}}\right\|_{2}$ s.t. $\left\|\boldsymbol{\alpha}_{\mathrm{i}}\right\|_{0} \leq c$.

5. Count the number of atoms from each $N$ sub-dictionary required to present $\boldsymbol{x}_{\mathbf{i}}$.

6. Weighting the selected atoms by their expansion coefficients. 
7. Calculate correlation of the sum of the weighted atoms from each $N$ sub-dictionary with input $\boldsymbol{x}_{\mathrm{i}}$.

We consider the average of correlation coefficients and number of atoms over the whole dataset as shown in table 2 .

Table 2: Number of selected weighted atoms and correlation coefficient for each sub-dictionary of different vanishing moment.

\begin{tabular}{|c|c|c|c|}
\hline Dictionary & N sub-dictionary & $\begin{array}{c}\text { No. } \\
\text { atoms }\end{array}$ & $\begin{array}{l}\text { Corr. } \\
\text { Coef. }\end{array}$ \\
\hline \multirow{7}{*}{$\mathrm{dbN}$} & $\mathrm{db} 2$ & 62 & 0.195 \\
\hline & $\mathrm{db} 3$ & 64 & 0.697 \\
\hline & $\mathrm{db} 4$ & 58 & 0.490 \\
\hline & $\mathrm{db} 5$ & 38 & 0.205 \\
\hline & $\mathrm{db} 6$ & 61 & 0.804 \\
\hline & $\mathrm{db} 7$ & 55 & 0.309 \\
\hline & $\mathrm{db} 8$ & 58 & 0.302 \\
\hline \multirow{7}{*}{ symN } & sym2 & 83 & 0.303 \\
\hline & sym3 & 64 & 0.651 \\
\hline & sym4 & 44 & 0.749 \\
\hline & sym5 & 85 & 0.280 \\
\hline & sym6 & 11 & 0.756 \\
\hline & sym7 & 71 & 0.426 \\
\hline & sym8 & 38 & 0.510 \\
\hline \multirow{5}{*}{ coifN } & coif1 & 94 & 0.832 \\
\hline & coif2 & 78 & 0.847 \\
\hline & coif3 & 64 & 0.393 \\
\hline & coif4 & 72 & 0.340 \\
\hline & coif5 & 87 & 0.338 \\
\hline
\end{tabular}

\subsection{Quantitative Performance analysis}

The quantitative performance of SDM in denoising ECG segment is evaluated using,

$$
S N R_{\text {imp }}[d B]=S N R_{\text {out }}-S N R_{\text {in }},
$$

where $S N R_{\text {in }}$ and $S N R_{\text {out }}$ are the signal to noise ratio at the input and output of the SDM model. Akaike's information criterion (AIC) is considered in the work to estimate the optimal number of nonzero coefficients $c$, which indicates the best SDM that has high $S N R_{\mathrm{imp}}$ and a few atoms [5],

$$
A I C=n \times \ln \left(\text { mean }\left(\operatorname{var}\left(x[i]-x^{\prime}[i]\right)\right)+2 \times c,\right.
$$

where $x[i]$ and $x^{\prime}[i]$ are the original and denoised ECG segment, respectively. For better interpretation, the weighted AIC is considered,

$$
A I C_{\text {weight }}=\exp \left(\frac{A I C_{\mathrm{min}-A I j}}{2}\right),
$$

where $A I C_{\min }$ is the minimum $A I C$ value in the searching range, and $A I C_{j}$ is the $j$-th AIC value of SDM model. The optimal SDM model has the highest $A I C_{\text {weight }}$ equal to 1 at specific number of atoms.

\section{Results}

After applying the algorithm in section 2.4 to the ECG segments mentioned in section 2.1, the sub-dictionaries with high correlation and possibly few atoms are considered to construct the optimal dictionary. From table 2, the proposed multi-wavelet type dictionary (MWD) is established from the following sub-dictionaries, namely coif1, coif2, db3, db6, sym4 and sym6. Moreover, the DCT dictionary is also considered to have smoother representation of periodic components, e.g., P-wave and T-wave (see Figure 2 blue). For evaluation, the corrupted ECG segments with simulated white noise of different input $S N R_{\text {in }}(10,20,30 \mathrm{~dB})$ are generated. The performance of the main three dictionaries and the optimal MWD dictionary in denoising ECG segments are evaluated in terms of number of atoms and the improvement of $S N R_{\mathrm{imp}}$. Akaike's information criterion is used to find the optimal number of atoms.

\begin{tabular}{|c|c|c|c|}
\hline$S N R_{\text {in }}$ & Dictionary & No. atoms & $S N R_{\text {imp }}$ \\
\hline \multirow{5}{*}{$10 \mathrm{~dB}$} & $\mathrm{dbN}$ & 30 & $3.21 \mathrm{~dB}$ \\
\hline & symN & 30 & $3.49 \mathrm{~dB}$ \\
\hline & coifN & 36 & $3.29 \mathrm{~dB}$ \\
\hline & MWD & 35 & $3.96 \mathrm{~dB}$ \\
\hline & MWD+DCT & 17 & $5.90 \mathrm{~dB}$ \\
\hline \multirow{5}{*}{$20 \mathrm{~dB}$} & $\mathrm{dbN}$ & 62 & $1.80 \mathrm{~dB}$ \\
\hline & symN & 57 & $2.02 \mathrm{~dB}$ \\
\hline & coifN & 59 & $1.92 \mathrm{~dB}$ \\
\hline & MWD & 56 & $3.29 \mathrm{~dB}$ \\
\hline & MWD+DCT & 33 & $3.62 \mathrm{~dB}$ \\
\hline \multirow{5}{*}{$30 \mathrm{~dB}$} & $\mathrm{dbN}$ & 116 & $0.04 \mathrm{~dB}$ \\
\hline & symN & 113 & $0.03 \mathrm{~dB}$ \\
\hline & coifN & 133 & $-0.07 \mathrm{~dB}$ \\
\hline & MWD & 93 & $0.71 \mathrm{~dB}$ \\
\hline & MWD+DCT & 62 & $1.87 \mathrm{~dB}$ \\
\hline
\end{tabular}

Table 3: The evaluation of different dictionary in terms of Number of atoms and improvement of $S N R_{\mathrm{imp}}$ for different $S N R_{\text {in }}$.

The $A I C_{\text {weight }}$ has estimated the optimal number of atoms successfully (see Figure 3). Table 3 shows that considering only one type of wavelet dictionary with different escape moments may not be the optimal choice. Establishing a dictionary from highly correlated sub-dictionaries of different mother wavelets, like the proposed MWD, leads to high improvement of SNR and better sparsity of SDM. For example, the results obtained for $S N R_{\text {in }}=30 \mathrm{~dB}$ show that the MWD has $S N R_{\mathrm{imp}}=0.71 \mathrm{~dB}$ and only required 93 atoms, while $\mathrm{dbN}$, symN, coifN dictionaries have $\mathrm{SNR}_{\mathrm{imp}}=0.04$, $0.03,-0.07 \mathrm{~dB}$ and $116,113,133$ atoms respectively. However, P-wave and T-wave are not well represented using 
only wavelet atoms of MWD (see Figure 2 black). Therefore, we proposed DCT as an additional sub-dictionary to the MWD to provide atoms which help to represent the low frequency parts like P-wave and T-wave more efficiently (see Figure 2 blue). Consequently, the additional DCT sub-dictionary has improved the sparsity of SDM significantly as well as the $S N R_{\text {imp }}$.
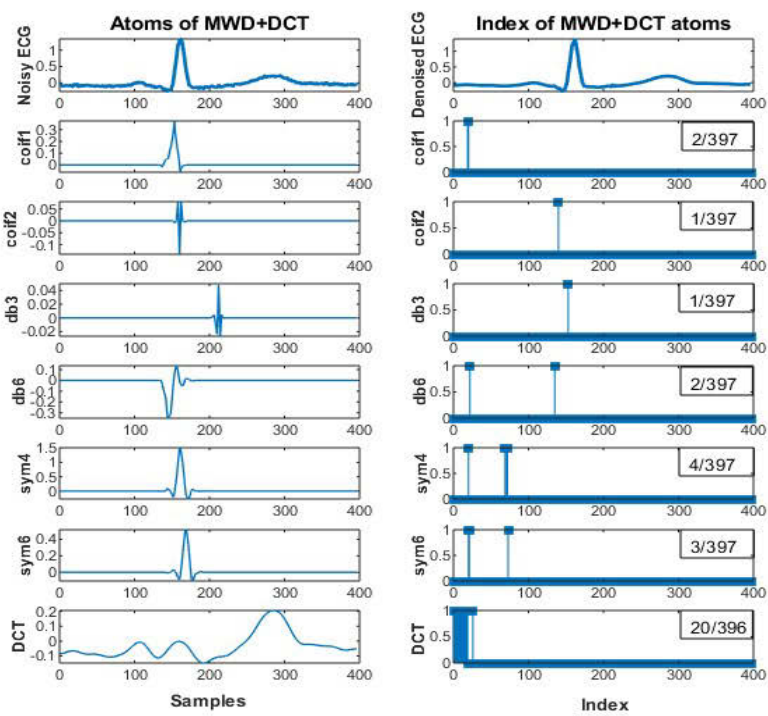

type. However, a predefined dictionary of only single-type wavelets atoms is less accurate in representing low frequency components of the ECG segment (like P-wave and T-wave) compared to our approach. It is possible to say that the optimal overcomplete dictionary should be constructed from highly correlated atoms of different wavelet types, plus DCT atoms to represent the target signal, e.g., ECG, sparsely.
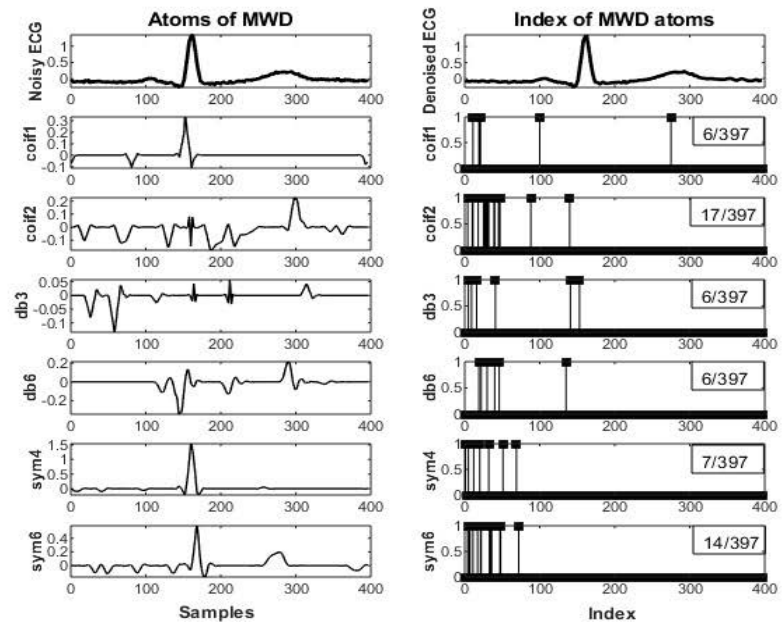

Figure 2: The sum of the selected atoms and its index in each sub-dictionary in case of $S N R_{\text {in }}=20 \mathrm{~dB}$ (blue) MWD+DCT, (black) MWD.
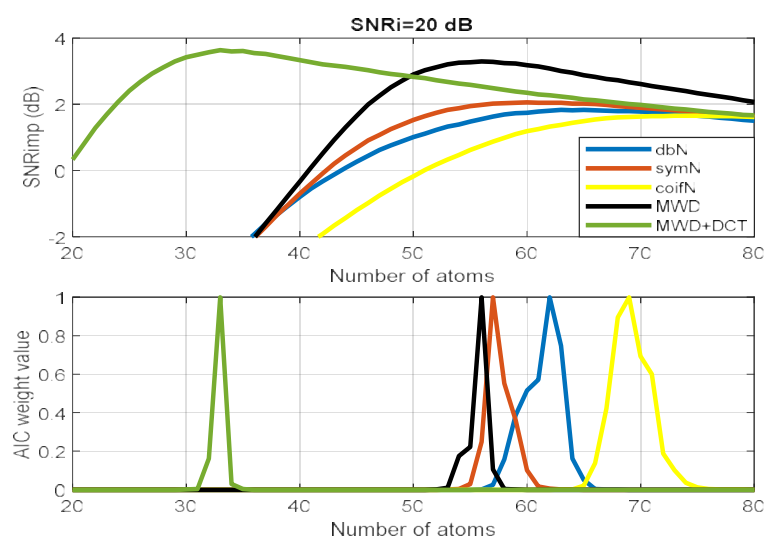

Figure 3: The performance of $\mathrm{dbN}$, symN, coifN, MWD, and MWD+DCT in term of (top) $S N R_{\text {imp }}$, (bottom) the $A I C_{\text {weight }}$ with respect to the number of atoms.

\section{Discussion and Conclusions}

The sparse modeling with loose sparsity-constrain has been successfully implemented in the work to find an optimal multiwavelet type dictionary to denoise ECG signal. This is achieved by considering the sub-dictionaries of highly correlated atoms to the target signal. The main conclusion is that multi-wavelet type dictionary can have better performance in term of $S N R_{\text {imp }}$ and sparsity with dictionary of one wavelet

\section{Author Statement}

Research funding: We would like to thank DAAD for supporting the $\mathrm{PhD}$ work of Fars Samann (Grand Reference: 57507871).

\section{References}

[1] Luengo D, Meltzer D, Trigano T. An Efficient Method to Signals. Applied Sciences. 2018;8:1-18.

[2] Rubinstein R, Bruckstein A, Elad M. Dictionaries for Sparse Representation Modeling. Proceedings of the IEEE. 2010;98:1045-1057.

[3] Černá D, Rebollo-Neira L. Construction of wavelet dictionaries for ECG modelling. MethodsX. 2021;8:1-30.

[4] Liu F, Liu C, Zhao L, Zhang X, Wu X, et al. An Open Access Database for Evaluating the Algorithms of Electrocardiogram Rhythm and Morphology Abnormality Detection. Journal of Medical Imaging and Health Informatics. 2018;8:368-1373.

[5] Samann F, Schanze T. On estimating the optimal autoencoder model for denoising ECG using Akaike Information Criterion. AUTOMED-Automation in Medical Engineering. 2021, Basel, Switzerland. In Zenodo. 2021. https://doi.org/10.5281/zenodo.4925814 Learn Overcomplete Multi-Scale Dictionaries of ECG 